Nota Científica

(Short communication)

\title{
NUEVO REGISTRO AL NORTE DE COLOMBIA DE LA SERPIENTE ALACRANERA, STENORRHINA DEGENHARDTII BERTHOLD 1846 (SQUAMATA: COLUBRIDAE), CON COMENTARIOS SOBRE SU COMPORTAMIENTO DEFENSIVO
}

\section{NEW RECORD IN NORTHERN COLOMBIA OF THE SCORPION-EATING SNAKE STENORRHINA DEGENHARDTII BERTHOLD 1846 (SQUAMATA: COLUBRIDAE), WITH COMMENTS ON ITS DEFENSIVE BEHAVIOR}

\author{
JULIO CESAR ACUÑA-VARGAS ${ }^{1}{ }^{*}$, YOELIS YEPES-PÉREZ ${ }^{1}$, KARICK JOTTY A. ${ }^{2}$ \\ ${ }^{1}$ Universidad de La Guajira, Grupo de investigación en Ecología, Biodiversidad en Ecosistemas Tropicales (Ebet), Facultad de \\ Ciencias Básicas y Aplicadas. Laboratorio de Ciencias Biológicas, Kilómetro 5 Vía a Maicao, Riohacha, La Guajira - Colombia. \\ <Yyepes@uniguajira.edu.co> \\ ${ }^{2}$ Universidad de Cartagena, Grupo de investigación en Fisiología y Neurociencia, Facultad de Ciencias Exactas y Naturales. \\ Cartagena, Bolívar - Colombia. Sede San Pablo, Zaragocilla. <Kjottya@unicartagena.edu.co> \\ *Autor de correspondencia: <Jcacuna@uniguajira.edu.co; Julioacunavargas@gmail.com>
}

Recibido: 14/08/ 2017; aceptado: 19/01/2018; publicado en línea: 23/10/2018

Editor responsable: Gustavo Aguirre

Acuña-Vargas, J. C., Yepes-Pérez, Y., Jotty-A., K. (2018) Nuevo registro al norte de Colombia de la serpiente alacranera, Stenorrhina degenhardtii Berthold 1846 (Squamata: Colubridae), con comentarios sobre su comportamiento defensivo. Acta Zoológica Mexicana (nueva serie), 34, 1-6. DOI: https://doi.org/10.21829/azm.2018.3412109

\begin{abstract}
A confirmed record of the Scorpion-eating Snake, Stenorrhina degenhardtii (Berthold 1846), is provided for the valleys between the Sierra Nevada of Santa Marta and Serrania de Perija in the municipality of El Molino, department of La Guajira. This record extends the geographic distribution of this species in the Caribbean Region, Northeast of Colombia. The information presented herein contributes to the knowledge of $S$. degenhardtii natural history and reptile diversity in the department of La Guajira, Colombia.
\end{abstract}

El género Stenorrhina (Duméril, 1853) lo representan dos especies, Stenorrhina degenhardtii (Berthold, 1846) y Stenorrhina freminvillei (Duméril, Bibrón \& Duméril, 1854), serpientes ovíparas con amplia distribución a través de México, Centro América y el norte de Suramérica (Köhler, 2003). La distribución de $S$. degenhardtii se extiende desde el Sureste de México hasta Perú, incluyendo el Noroeste de Venezuela y oeste de Ecuador, desde el nivel del mar hasta los 2,750 m (Pérez-Santos \& Moreno, 1988; Wallach et al., 2014). El presente registro de S. degenhardtii es el primer referente confirmado sobre la presencia de la especie para el departamento de La Guajira en Colombia que permite adicionalmente aportar información sobre aspectos de su comportamiento defensivo.

El ejemplar de S. degenhardtii se encontró en el municipio El Molino localizado en el sur de La Guajira, llanura entre la Serranía de Perijá y La Sierra Nevada de Santa Marta en el departamento de La 
Guajira, Colombia, escenario de una mezcla de coberturas de la tierra naturales y antropogénicas que se extienden desde los 150 a los 2,200 msnm (Alcaldía municipal El Molino, 2000).

Desde el año 2009 se han realizado visitas periódicas a esta localidad, con el fin de conocer la diversidad de fauna silvestre presente. Los monitoreos biológicos por parte de estudiantes del programa de Biología de La Universidad de La Guajira en el año 2014 permitieron el encuentro de un ejemplar de $S$. degenhardtii, en el área urbana del municipio asociada al colegio Ismael Rodríguez Fuentes (10³9’17', N, 72 55’ 53', ' W; 242 msnm), lugar del campamento de estudio (Fig. 1).

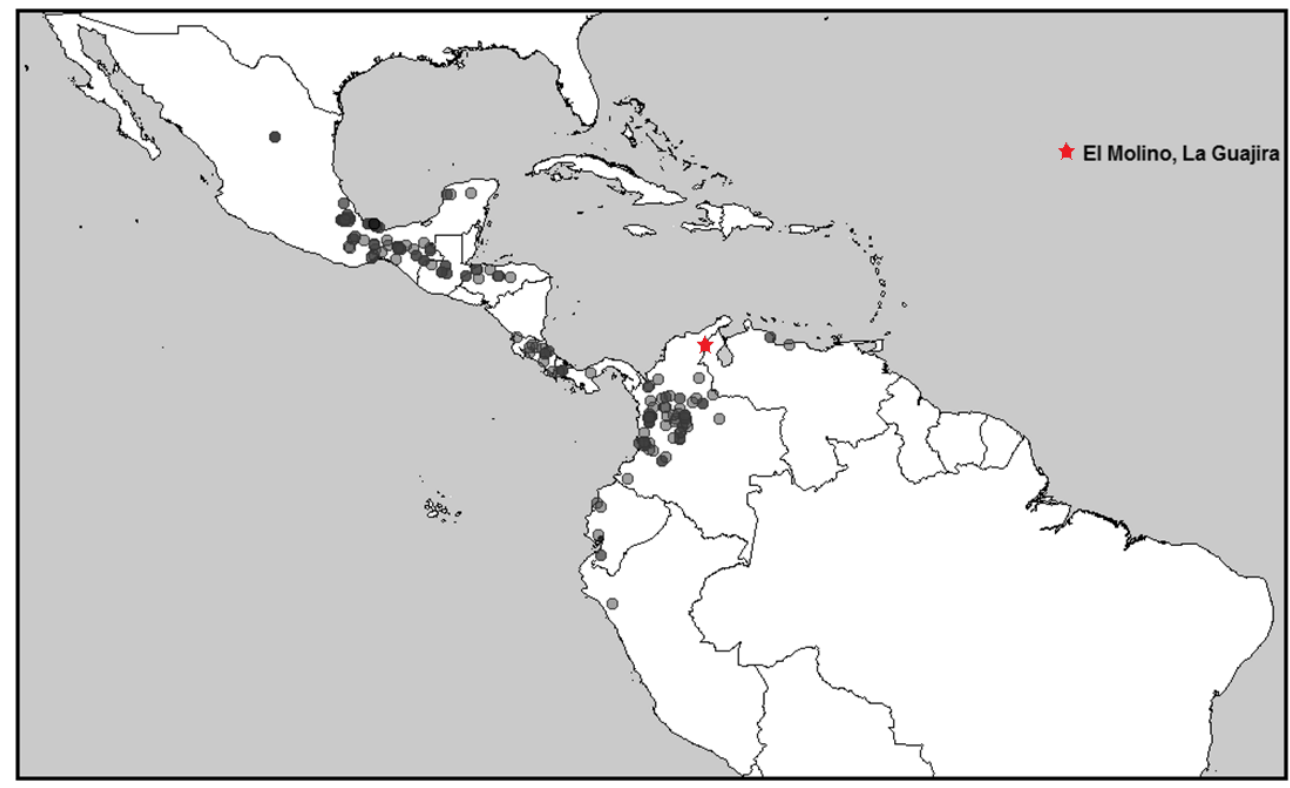

Figura 1. Mapa de distribución de Stenorrhina degenhardtii (Berthold 1846) según registros GBIF y localidad de encuentro en el municipio El Molino, La Guajira, Colombia.

El encuentro de $S$. degenhardtii, ocurrió durante el monitoreo de fauna silvestre sobre transectos al azar, entre las 06:00 y 12:00 hrs. del 19 de octubre de 2014. La serpiente se observó durante 60 minutos desde el momento de encuentro (09:20 hrs.), mientras se desplazaba y sin ser manipulada. A continuación, se capturó y se observó el comportamiento del individuo en respuesta al acercamiento del humano y a la manipulación. Después de la observación, se realizó la determinación taxonómica con base en las descripciones de Pérez-Santos y Moreno (1988), Köhler (2003) y Navarrete et al. (2005), posteriormente se liberó en el sitio de encuentro.

El ejemplar capturado fue una hembra que presentó condición corporal normal, escamado limpio, sin presencia aparente de ectoparásitos, con longitud total de $40.91 \mathrm{~cm}$ que reúne las características claves de la especie $S$. degenhardtii.

Al no ser manipulada, la serpiente exhibió movimientos sutiles y lentos en su desplazamiento, lo que le permite confundirse con su entorno, este comportamiento cambia cuando la serpiente se enfrenta a algún tipo de disturbio. En el individuo capturado se observaron tres tipos de comportamientos de huida, los cuales se describen como comportamiento de huida defensiva, desplazamiento pausado (Huida 1; Cuadro 1) comportamiento de huida defensiva, desplazamiento continuo (Huida 2; Cuadro 2), comportamiento de huida defensiva desplazamiento continuo durante la manipulación o "captura” (Huida 
3; Cuadro 3). Un cuarto patrón de comportamiento “defensivo” de la serpiente alacranera se observa cuando

Cuadro 1. Descripción de comportamiento de Huida defensiva 1, desplazamiento pausado, para ejemplar de Stenorrhina degenhardtii (+) municipio el Molino, La Guajira, Colombia. Segundos (s).

\begin{tabular}{|c|c|c|c|}
\hline Comportamiento & Descripción & Latencia (s) & Duración (s) \\
\hline Desplazamiento 1 & $\begin{array}{l}\text { Se presenta cuando el animal aparentemente se encuentra } \\
\text { sin advertir la presencia del humano; movimientos } \\
\text { sigilosos, lentos y rectilíneos que se producen antes de } \\
\text { ingresar a la zona de detección de la amenaza (Lynch, } \\
\text { 2012). }\end{array}$ & 0 & 60 \\
\hline Desplazamiento 2 & $\begin{array}{l}\text { Se presenta una vez el animal se encuentra frente al } \\
\text { disturbio y cuando éste ingresa a la zona de detección de } \\
\text { la amenaza (Lynch, 2012). Se caracteriza por } \\
\text { movimientos rápidos y serpenteantes. }\end{array}$ & 0 & 300 \\
\hline Pausa en alerta 1 & $\begin{array}{l}\text { Se presenta durante el desplazamiento } 2 \text {, el animal } \\
\text { detiene su desplazamiento, o devuelve el primer tercio del } \\
\text { cuerpo. Este comportamiento disminuye en la medida que } \\
\text { el animal se aleja del foco de disturbio y permanece el } \\
\text { desplazamiento } 2 \text {. }\end{array}$ & $\begin{array}{l}28 \\
17 \\
13\end{array}$ & $\begin{array}{l}6 \\
2 \\
3\end{array}$ \\
\hline
\end{tabular}

Cuadro 2. Descripción de comportamiento de Huida defensiva 2, desplazamiento continuo, para ejemplar de Stenorrhina degenhardtii (ㅇ) municipio el Molino, La Guajira, Colombia. Segundos (s).

\begin{tabular}{llcc}
\hline Comportamiento & \multicolumn{1}{c}{ Descripción } & Latencia (s) & Duración (s) \\
\hline Desplazamiento 3 & Se presenta una vez el animal se encuentra frente al & 0 & 300 \\
& $\begin{array}{l}\text { disturbio, se produce cuando se ingresa a la zona de } \\
\text { defensa o seguridad (Lynch, 2012). Se caracteriza por } \\
\\
\text { movimientos rápidos, serpenteantes y continuos. }\end{array}$ & \\
\hline
\end{tabular}

Cuadro 3. Descripción de comportamiento de Huida defensiva 3 (manipulación o captura), desplazamiento continuo, por acercamiento de amenaza, ingreso a zona de seguridad, para Stenorrhina degenhardtii (+) municipio el Molino, La Guajira, Colombia. Segundos (s).

\begin{tabular}{llcc}
\hline Comportamiento & \multicolumn{1}{c}{ Descripción } & Latencia (s) & Duración (s) \\
\hline Desplazamiento 4 & $\begin{array}{l}\text { Se presenta una vez el animal se encuentra frente al } \\
\text { disturbio y es manipulado, se produce cuando se ingresa } \\
\end{array}$ & 0 & 300 \\
& a la zona de defensa o seguridad (Lynch 2012). Se & & \\
& caracteriza por movimientos rápidos, escurridizos y & & \\
& continuos. El animal evita las zonas abiertas. & & \\
\hline Defensa 1 & Posteriormente al desplazamiento 4, el animal aumenta & 0 & 3 \\
& o disminuye el grosor corporal usando el movimiento & 25 & 5 \\
& de las costillas del medio del cuerpo, se presenta cuando & 32 & 3 \\
& el animal es manipulado y se ejerce presión sobre el & 43 & 2 \\
& cuerpo del animal. & & \\
\hline
\end{tabular}


la serpiente no es manipulada (Cuadro 4), pero se encuentra expectante, busca permanecer alejada de espacios abiertos. Cuando el ejemplar se siente amenazado, rápidamente devuelve su desplazamiento, el primer tercio del cuerpo se devuelve a la mitad del mismo sobre el cuerpo y el último tercio se dirige rápidamente hacia adelante (Fig. 2a,b), y se observó que el animal busca ocultar la cabeza bajo el cuerpo para su protección con el mismo (Fig. 2c, Cuadro 4), de manera similar al comportamiento presentado por otras serpientes como las corales verdaderas (Micrurus spp.); después de estos movimientos la serpiente buscó desenvolverse de manera lenta, pero esta vez tendió a presentar el primer tercio del cuerpo más cercano al resto del cuerpo y permaneció en una condición de alerta (Fig. 2d).

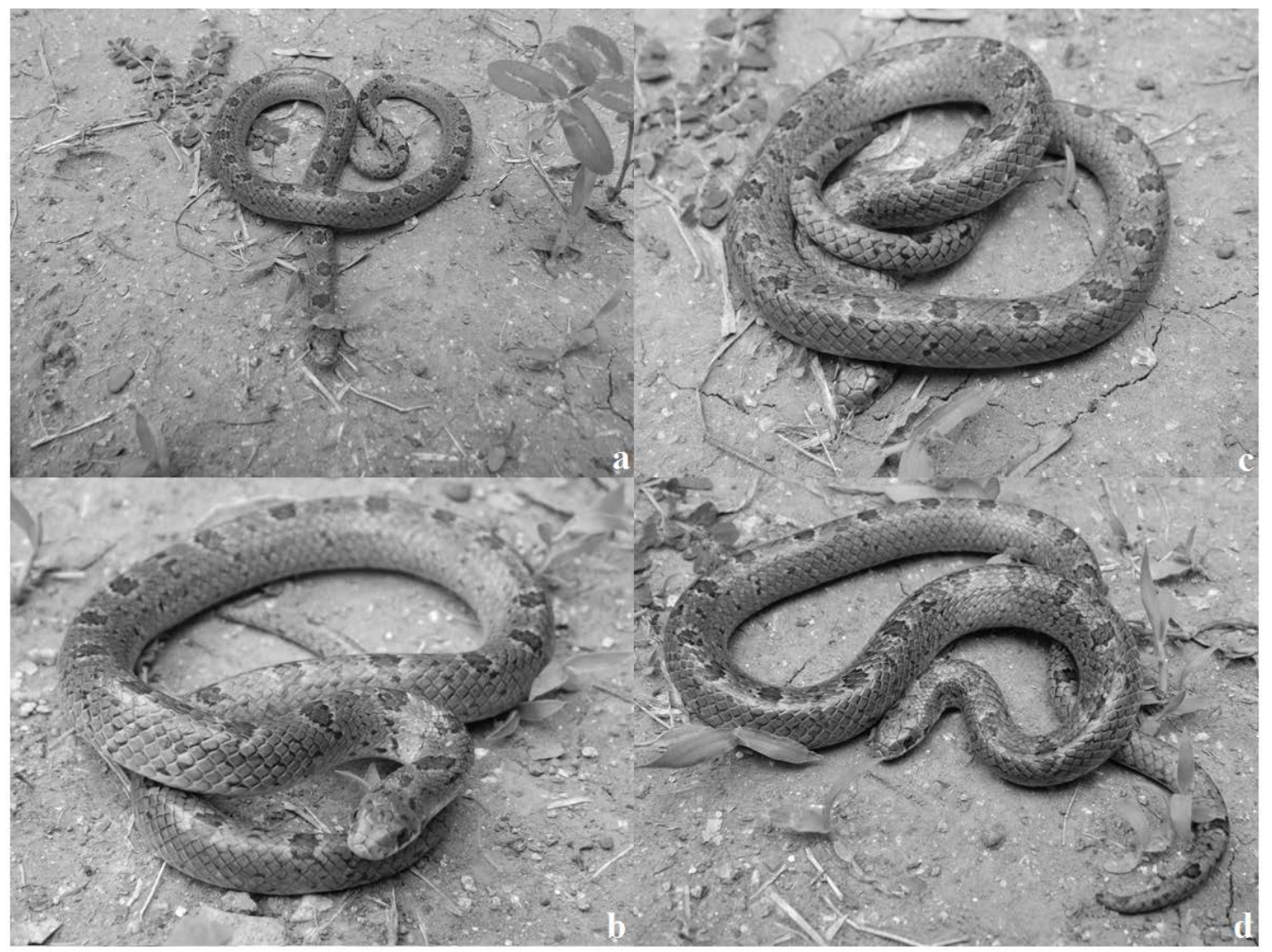

Figura 2. Comportamiento defensivo en Stenorrhina degenhardtii ( + ), encontrada en el municipio El Molino, La Guajira. (a) Pausa en alerta 3; (b) Pausa en alerta 1; (c) Pausa en alerta 3; (d) Pausa en alerta 2.

El presente registro extiende la distribución de $S$. degenhardtii al noreste de Colombia, los registros confirmados más cercanos de la especie se encuentran a más de $250 \mathrm{~km}$ de la localidad de estudio, correspondiente al departamento de Sucre y el departamento de Norte de Santander en Colombia y el estado del Táchira en la República Bolivariana de Venezuela (Navarrete et al., 2005).

Las serpientes son animales poco estudiados en la región Caribe de Colombia, en donde se han registrado aproximadamente 45 especies (Lynch, 2012). Su mención en documentos científicos se limita a listados de reptiles y muy rara vez a estudios directos sobre el grupo en la región Caribe (Acuña-Vargas \& Escorcia, 2005; Dávila \& Buelvas, 2009; Bermúdez-Guerrero, 2010; Ruiz, 2012; Blanco et al., 2013; Acuña-Vargas et al., 2015; Márquez \& Gómez, 2015; Acuña-Vargas, 2016; Cuellar et al., 2016) y se 
desconocen aspectos relacionados con la historia natural de las especies, comportamiento, papel ecológico en los ecosistemas y estado de conservación actual.

Cuadro 4. Descripción de comportamiento defensivo por acercamiento de amenaza, ingreso a zona de seguridad, para Stenorrhina degenhardtii (q) municipio el Molino, La Guajira, Colombia. Segundos (s), ${ }^{*}$ ) tiempo de latencia en segundos, después de presentarse el estímulo (movimientos rápidos y agresivos).

\begin{tabular}{llcc}
\hline Comportamiento & \multicolumn{1}{c}{ Descripción } & Latencia (s) & Duración (s) \\
\hline Pausa en alerta 2 & Se presentan durante el desplazamiento 4, el animal & $13\left(0^{*}\right)$ & 2 \\
& detiene su desplazamiento, simplemente deteniendo el & $25\left(0^{*}\right)$ & 1 \\
& movimiento de desplazamiento o devolviendo el primer & $32\left(0^{*}\right)$ & 1 \\
& tercio del cuerpo. Este comportamiento se presenta cada & $37\left(0^{*}\right)$ & 1 \\
& vez que el animal advierte la presencia del foco de & $43\left(0^{*}\right)$ & 1 \\
& disturbio y éste presenta movimientos rápidos y & $53\left(0^{*}\right)$ & 1 \\
& agresivos cercanos a la serpiente. & $63\left(0^{*}\right)$ & 1 \\
\hline Pausa en alerta 3 & Se presentan durante el desplazamiento 4, cuando el & $70\left(0^{*}\right)$ & 1 \\
& animal se encuentra en alerta, detiene su desplazamiento, & $90\left(0^{*}\right)$ & 6 \\
& este comportamiento se presenta cada vez que el animal & $120\left(0^{*}\right)$ & 13 \\
& advierte la presencia del foco de disturbio y se le & $160\left(0^{*}\right)$ & 17 \\
& presentan movimientos rápidos y agresivos por parte del & $210\left(0^{*}\right)$ & 8 \\
& foco de disturbio muy cercanos a la serpiente. La & $250\left(0^{*}\right)$ & 10 \\
& serpiente detiene el movimiento de manera inmediata, & $270\left(0^{*}\right)$ & 14 \\
& devolviendo el primer y último tercio del cuerpo, y busca & $300\left(0^{*}\right)$ & 9 \\
& proteger la cabeza con el cuerpo. & & \\
\hline
\end{tabular}

Referentes sobre aspectos comportamentales en serpientes han venido incrementando (Ford, 1995), pero en Colombia los estudios son escasos. El ejemplar de S. degenhardtii observado se alejó antes de demostrar un comportamiento agresivo. La zona de detección (Lynch, 2012) se encuentra en una distancia aproximada de $1 \mathrm{~m}$, y en este campo de acción el animal siempre trata de huir durante la observación; la zona de defensa o seguridad (Lynch, 2012) es aproximadamente $0.5 \mathrm{~m}$ y en este campo de acción, el animal tiende a presentar movimientos más rápidos y defensivos, pero sin llegar a la agresión aparente. Este comportamiento se puede considerar como tolerante a la manipulación, a diferencia del mostrado por otras especies de serpientes de la misma familia que se tornan agresivas y de riesgo por mordedura desde el inicio de la manipulación, como es el caso de Leptophis ahaetulla (Linnaeus, 1758), Chironius carinatus (Linnaeus, 1758) y Thamnodynastes gambotensis (Pérez-Santos \& Moreno, 1989), entre otras (observación personal).

AgRADECIMIENTOS. Este aporte se realiza gracias al apoyo de la Universidad de La Guajira, en el marco de los proyectos Biodiversidad asociada a Bosque seco tropical y Museo de la diversidad biológica de La Guajira. Se extienden agradecimientos a los estudiantes del V semestre (2014-II) cursantes de las asignaturas Etología y métodos de campo II de la Universidad de La Guajira, por su compañía y colaboración en campo, a la Universidad de La Guajira por su apoyo logístico y económico, a la Institución técnica educativa agropecuaria Ismael Rodríguez Fuentes del Molino, por el apoyo de su personal docente, administrativo y operativo, en aspectos logísticos, locativos y por su constante colaboración en el proceso de dar a conocer la biota de la localidad de estudio; a los estudiantes miembros del grupo EBET por su colaboración en campo, al laboratorio de Ciencias Biológicas y la iniciativa de Colecciones Biológicas de la Universidad de La Guajira y de manera especial a Nicole, Julio y Paola por su apoyo y tiempo para este documento, además se extienden agradecimientos a diferentes amigos y colegas por algunos registros y comentarios aportados para este manuscrito, Jhon Calderón, Cristhian Murillo, Estefanía Silvera, Miguel Gutiérrez, Luis Arrieta. 


\section{LITERATURA CITADA}

Acuña-Vargas, J. C. (2016) Anfibios y reptiles asociados a cinco coberturas de la tierra, municipio de Dibulla, La Guajira, Colombia. Acta Zoológica Mexicana (nueva serie), 32, 133-146.

Acuña-Vargas, J. C., Yepes-Pérez, Y., Bastidas M. B. (2015) Anfibios y reptiles asociados a Bosque seco tropical en dos localidades del departamento de La Guajira, Colombia. Revista Ciencia e Ingeniería, 2, 1-10.

Acuña-Vargas, J. C., Escorcia H. J. E. (2005) Estimación poblacional para la especie Bothrops asper (Garman 1884) en los relictos de Bosque seco tropical alternohígrico presentes en el centro occidente del departamento del Atlántico. Tesis de pregrado. Universidad del Atlántico, Barranquilla, 133 pp.

Alcaldía Municipal El Molino. (2000) Esquema de ordenamiento territorial, municipio El Molino, La Guajira. El Molino, La Guajira, Colombia, 190 pp. Disponible In: http://cdim.esap.edu.co/BancoConocimiento/E/el_molino_-_la_guajira_-_eot_-_2000/el_molino__la_guajira_-_eot_-_2000.asp (Fecha de consulta: agosto 2017).

Bermúdez-Guerrero, F. (2010) Caracterización epidemiológica de los accidentes ofídicos, en pacientes pediátricos, Cartagena de Indias 2006-2007. Revista Ciencias Biomédicas, 1, 23-29.

Blanco T. A., Báez, L., Patiño-Flores, E., Renjifo, J. M. (2013) Herpetofauna del valle medio del río Ranchería, La Guajira, Colombia. Revista Biodiversidad Neotropical, 3, 113-122.

Cuellar Gordo, L. C., Amador Orozco, B., Olivares Goenaga, G., Borré Ortiz, Y. M., Pinedo Otálvaro, J. (2016) Comportamiento epidemiológico del accidente ofídico en el Departamento del Magdalena, Colombia (2009-2013). Revista Ciencias de la Salud, 14, 161-177.

Dávila, J., Buelvas, J. (2009) Reporte de algunas especies de ofidios presentes en el departamento de Sucre, Colombia. Revista Colombiana de Ciencia Animal-RECIA, 1, 273-278.

Ford, N. B. (1995) Experimental design in studies of snake behavior. Herpetological Monographs, 9, 130-139.

Köhler, G. G. K. (2003) Reptiles of Central America. Herpeton. Offenback, Germany. 367 pp.

Lynch, J. D. (2012) El contexto de las serpientes de Colombia con un análisis de las amenazas en contra de su conservación. Revista de la Academia Colombiana de Ciencias Exactas, Físicas y Naturales, 36, 435-449.

Márquez Gómez, M. A., Gómez Díaz, G. M. (2015) Ophidic accident in the department of Sucre, Colombia. Nova, 13, 39-46.

Navarrete, L. F., Rodriguez-Acosta, A., Contreras, Y., Briceño, J. M. (2005) Stenorrhina degenhardtii ocellata. JAN, 1876 in Venezuela. Herpetozoa, 18, 89-91.

Pérez-Santos, C., Moreno, A. G. (1988) Serpientes de Colombia. Museo Regionale di Scienze Naturali, Torino, Monografie XI. Madrid, España, 497 pp.

Ruiz, P. (2012) Reporte de las serpientes del municipio de Tamalameque, Cesar-Colombia. Revista MVZ Córdoba, 17, 2924-2927.

Wallach, V., Williams, K. L., Boundy, J. (2014) Snakes of the world: a catalogue of living and extinct species. CRC Press, 1227 pp. 